\title{
Detection of primary photons using Čerenkov imaging and surface detectors
}

\author{
Fausto Casaburo \\ University "La Sapienza" of Rome
}

\begin{abstract}
Discovered by Hess during some experiments about air ionization, cosmic rays are constituted by particles coming from the space. In the past, cosmic rays allowed the development of Particle Physics; indeed, thanks to their high energy not achievable in laboratories, they enabled new particles discovery. Today, interest about this radiation concerns both Astrophysics and Particle Physics. Indeed on the one hand, their knowledge allows formulation about new models of Universe structure and evolution or to acquire new knowledge about final objects of stars evolution; on the other hand cosmic rays allows us to study fundamental processes, as for example acceleration and interaction mechanisms of particles at energies not achievable in laboratories. Although it has passed more than a century after their discovery, there are many questions to which it isn't possible to answer yet or to which there isn't certainty about formulated theories. Some examples are about objects that can accelerate particles to high energy and acceleration mechanisms; indeed, even if there are some theories, we don't have experimental certainty. Moreover, although measured in many experiments, energy spectrum shows, especially in the region called "Knee", some differences between measuring made by experiments. Since magnetic fields deflect charged particles, their observation doesn't allow to go back to the source, so in cosmic rays study it's very important $\gamma$ rays observation because they aren't deflected by magnetic fields. In 1989 Whipple experiment allowed to observe, for the first time, $\mathrm{TeV}$ energy $\gamma$ rays coming from Crab Nebula. Thanks to many experiments made to answer questions about cosmic rays, more than 100 Very High Energy (VHE) $\gamma$ rays sources were observed since then; 60 out of 100 have galactic origin, as for instance Supernova Remnants or Pulsars; for the rest, apart from those not identified, they have extra-galactic origin. In this work some simulations, made by National Nuclear Physics Institute (INFN) subdivision of Torino (Italy) using Cosmic Ray SImulations for KAscade (CORSIKA), were analyzed to study differences between Extensive Air Shower (EAS) produced by $\gamma$ rays and EAS produced by protons.
\end{abstract}

35th International Cosmic Ray Conference - ICRC2017

10-20 July, 2017

Bexco, Busan, Korea 


\section{Introduction}

$\mathrm{L}$

Low energy cosmic rays, can be detected by detectors putted on satellites; instead, since their very low flux, high energy comsic rays, must be studied by ground experiments, observing showers of secondary particles, calledEAS, produced in atmosphere by interaction between cosmic rays and the atmosphere. In this perspective, to study $\gamma$ rays, the Large High Altitude Air Shower Observatory (LHAASO) experiment (Fig 1) is currently in planning phase; it will be composed by LHAASO- one Kilometer square extensive air shower Array (LHAASO-KM2A) (it will be composed by Electron Detector $(\underline{E D})$ and Muon Detector $(\overline{M D})$ to measure number and arrival time of particles, LHAASO- Water Čerenkov Detector Array (LHAASO-WCDA) a water Čerenkov detector to study cosmic rays of energies higher than $1 \mathrm{TeV}$ and LHAASO- Wide Field of view Čerenkov Telescope Array (LHAASO-WFCTA) a Čerenkov telescope system to measure longitudinal development of cosmic rays and to obtain information about primary cosmic rays. After the building at Daochen in China, LHAASO will allow to study the "High Energy Universe", allowing observation of $\gamma$ rays of energies in the range $300 \mathrm{GeV} \div 1 \mathrm{PeV}$ observing secondary particles of showers called $\boldsymbol{E A S}$, result of interaction between primary particles and atmosphere. One other important experiment, currently in planning phase, it's Čerenkov Telescope

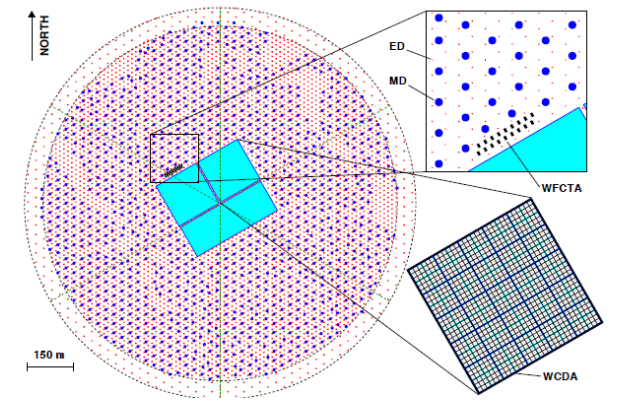

Figure 1: LHAASO outline.

Array (CTA (Fig 2). It will be built in two sites, at La Palma in Spain and at Parana in Chile. It will be the biggest Čerenkov imaging telescope array built so far and, although using different kind of detectors, CTA final goals are the same of LHAASO, Improving instrumentation respect to current and past experiments, they will allow observations not possibile up to now and they will improve results as well. To allow observation of $\gamma$ rays of energies in the range $20 \mathrm{GeV} \div 300 \mathrm{TeV}$, CTA will be composed by three kind of telescopes, the Large Size Telescope (LST) to make observations in the range $20 \mathrm{GeV} \div 100 \mathrm{GeV}$, the Medium Size Telescope (MST) for observations in the range $100 \mathrm{GeV} \div 10 \mathrm{TeV}$ and the Small Size Telescope (SST) for observations in the range $10 \mathrm{TeV} \div 300 \mathrm{TeV}$. Although LHAASO and CTA will have same final goals, since they will have different detectors, they will offer distinct opportunities to Astroparticle Physics; for example, LHAASO will have a better resolution at energies higher than $30 \mathrm{TeV}$ and it will allow observation of high section of the sky, CTA at energies approximately $1 \mathrm{TeV}$ and focused about single source.

\section{Simulations}

Thanks to specific simulation software it's possible to simulate EAS on the basis of theoretical 


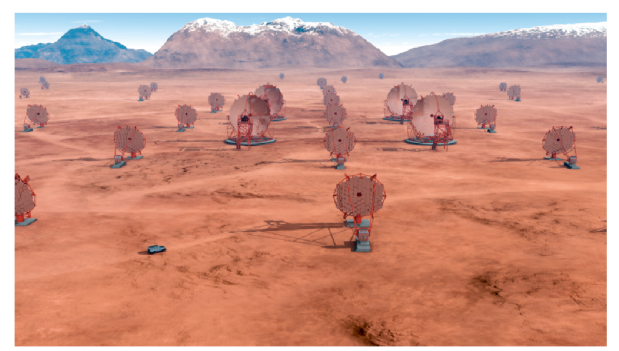

Figure 2: CTA artist's impression.

models and to use simulations both to study detector performances during fulfillment phase and to compare simulation results to experimental data in order to prove models during detector data acquisition; one of these software is, for example, CORSIKA Since in $\gamma$ astronomy experiments it's very important adrons rejection, some simulations made by CORSIKA were analyzed to compare EAS induced by protons in the range $1 \mathrm{GeV} \div 1000 \mathrm{GeV}$ and power law $\frac{d N}{d E} \propto E^{-2}$ (Fig 3 ) to EAS induced by $\gamma$ in the same energetic range and same power law (Fig 4). First of all, it was

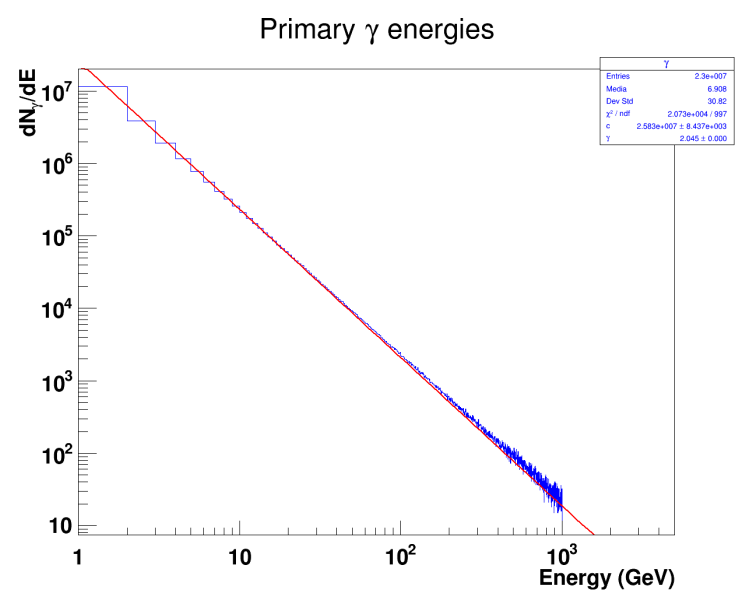

Figure 3: Photons simulated energy spectrum.

studied first interaction height of primary particles (Fig 5) showing that, due to different values of radiation length about electromagnetic showers and interaction length about adronic showers, $\gamma$ rays interact previous to protons in atmosphere; in addition, by calculating mean values of first interaction heights in the ranges $[20 \mathrm{GeV} \div 50 \mathrm{GeV}],[50 \mathrm{GeV} \div 100 \mathrm{GeV}],[100 \mathrm{GeV} \div 200 \mathrm{GeV}]$, $[200 \mathrm{GeV} \div 350 \mathrm{GeV}],[350 \mathrm{GeV} \div 600 \mathrm{GeV}]$ and $[600 \mathrm{GeV} \div 1000 \mathrm{GeV}]$ it was showed that first interaction height of gamma rays is almost constant in energy; instead, due to $p p$ cross section (Fig 6), protons first interaction height mean values are a bit higher for energies $E<100 \mathrm{GeV}$ than for higher energies.

\section{Results}

Our concentration was focused about EAS to study differences between the showers on the basis of primary particle. For this reason, first of all, arbitrarily choosing two primary particles 


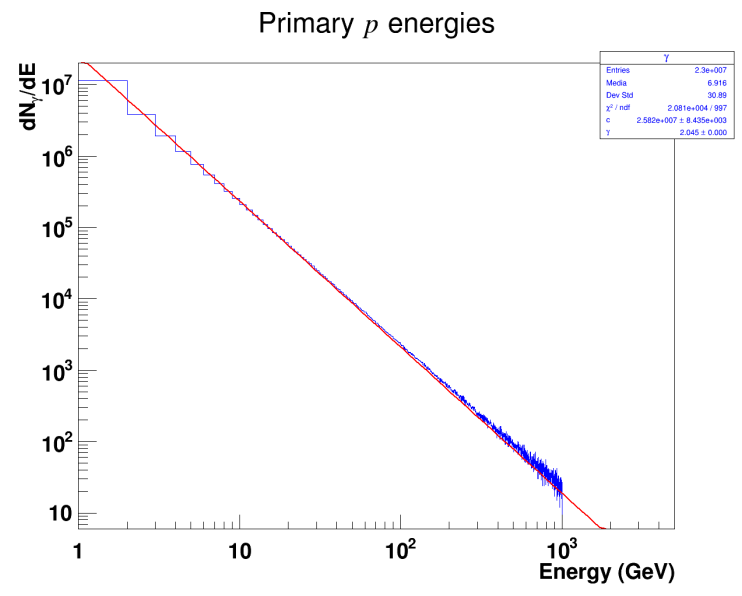

Figure 4: Protons simulated energy spectrum.
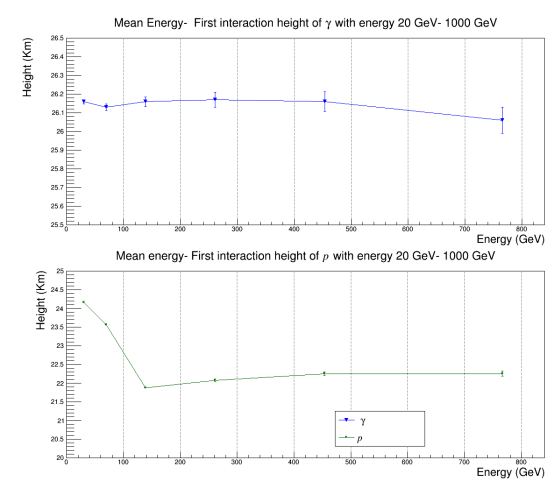

Figure 5: Height of first interaction mean values.

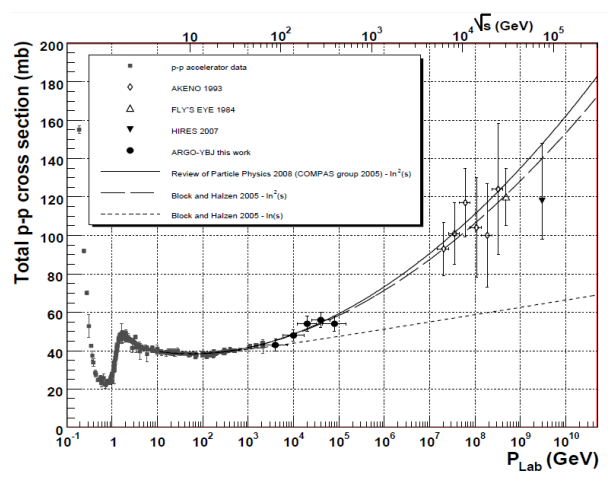

Figure 6: $p p$ cross section from http://arxiv.org/pdf/0904.4198v1.pdf.

energies $E \sim 150 \mathrm{GeV}$ and $E \sim 1 \mathrm{TeV}$, it was plotted secondary particles distributions at observation level (setted on $4300 \mathrm{~m}$ above sea level); by plots we can see that secondary particles of $\gamma$ rays (Fig 7) showers are arranged on surfaces centered in EAS core smaller than particles of proton showers (Fig 8). Later it was observed the difference between number of secondaries $e^{ \pm}$and $\mu^{ \pm}$produced by $\gamma$ rays showers (Fig 9 ) and proton showers (Fig 10 ; ; by plots it's clear 


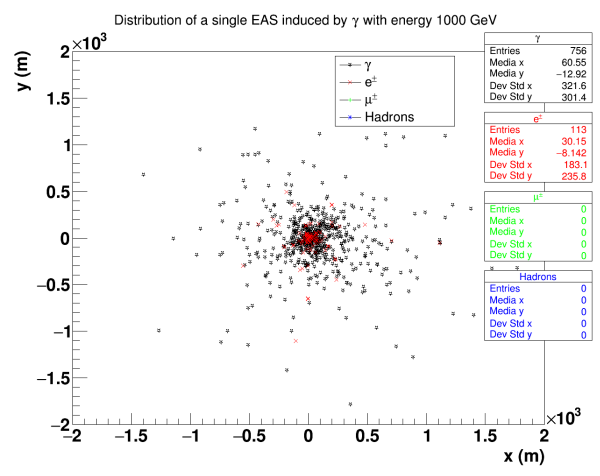

Figure 7: Distribution at observation level of EAS induced by $\gamma$ of energy $E \sim 1 \mathrm{TeV}$.

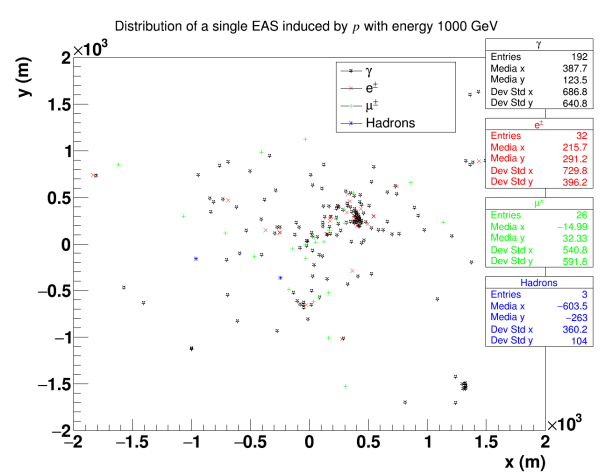

Figure 8: Distribution at observation level of EAS induced by $p$ of energy $E \sim 1 \mathrm{TeV}$.

that $\frac{e^{ \pm}}{\mu^{ \pm}}$ratio is higher for $\gamma$ rays showers than for proton showers, because in proton showers we have higher $\mu^{ \pm}$than in $\gamma$ rays showers. Additionally, calculating $e^{ \pm}$and $\mu^{ \pm}$mean values in

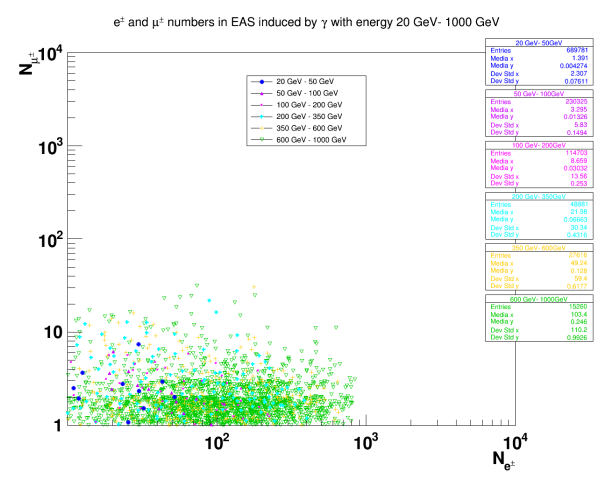

Figure 9: Number of $e^{ \pm}$and $\mu^{ \pm}$produced in EAS induced by primary $\gamma$.

energy ranges (Fig.11), it was showed their increasing production for higher primary particles energies. Very important is the difference about lateral development of the EAS; indeed, to confirm what showed by plots about distributions at observation level, it was calculated particles density in circular crowns centered in the EAS core up to $10 \mathrm{Km}$ from it. Plots showed that, increasing distance from core, density decreasing of secondary particles produced by $\gamma$ rays showers (Fig, 12) 


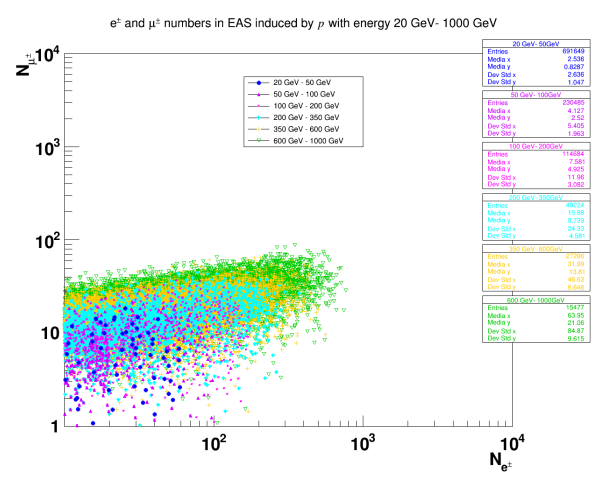

Figure 10: Number of $e^{ \pm}$and $\mu^{ \pm}$produced in EAS induced by primary $p$.

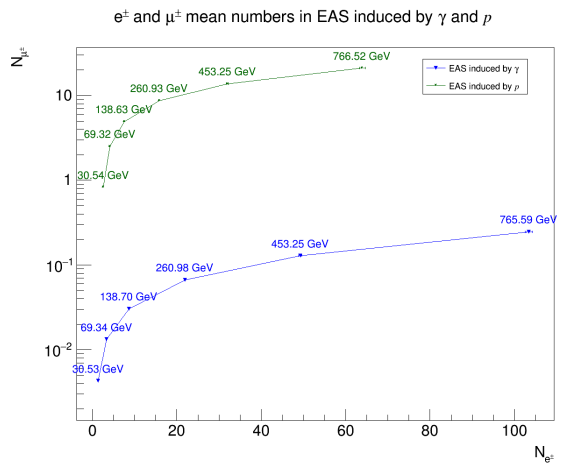

Figure 11: Mean value of $e^{ \pm}$and $\mu^{ \pm}$produced in EAS induced by primary $p$ in function of primary particles energy.

is faster than secondary particles produced by proton showers (Fig 13), so probability to find particles of $\gamma$ rays showers faraway from the core is smaller than to find particles of proton showers. Lastly, arbitrarily choosing 3 distances from the core $10 \mathrm{~m}, 100 \mathrm{~m}$ and $600 \mathrm{~m}$ it was calculated

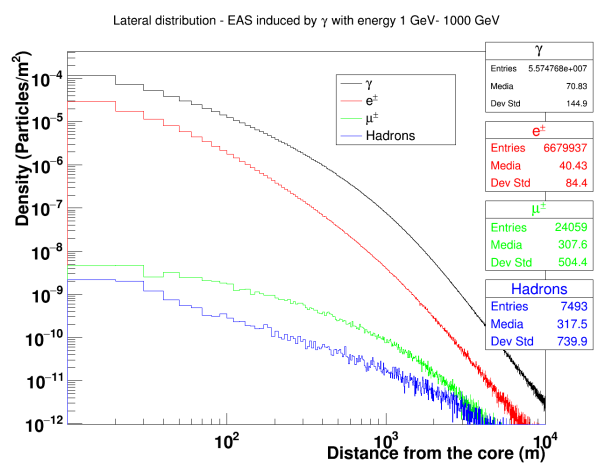

Figure 12: Lateral distribution of EAS induced by primary $\gamma$.

particles density for energy ranges of primary particles $[20 \mathrm{GeV} \div 50 \mathrm{GeV}],[50 \mathrm{GeV} \div 100 \mathrm{GeV}]$, $[100 \mathrm{GeV} \div 200 \mathrm{GeV}],[200 \mathrm{GeV} \div 350 \mathrm{GeV}],[350 \mathrm{GeV} \div 600 \mathrm{GeV}]$ and $[600 \mathrm{GeV} \div 1000 \mathrm{GeV}]$; as result (Fig 14 and Fig 15 it's clear that for fixed distances, increasing primary particles energy, secondary particles density increases too. 


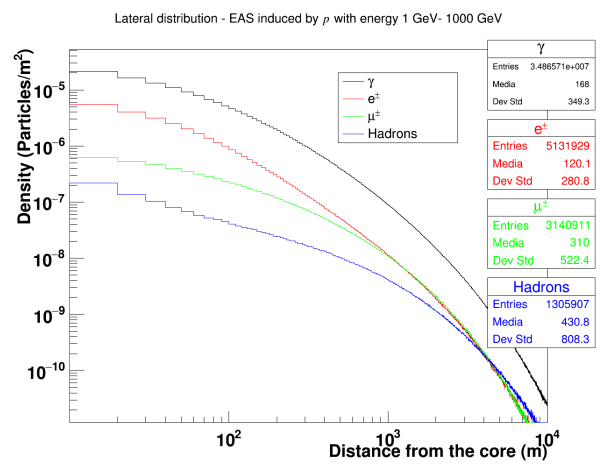

Figure 13: Lateral distribution of EAS induced by primary $p$.

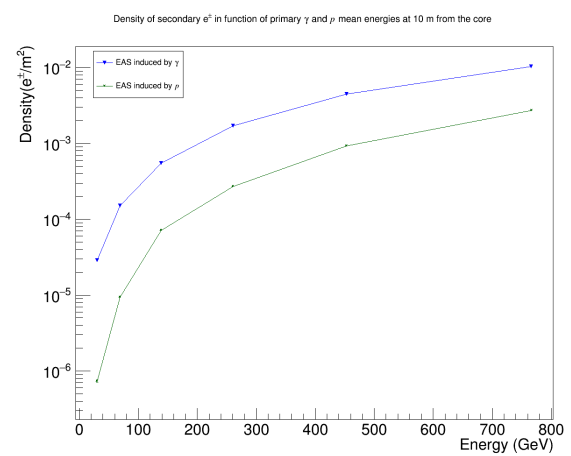

Figure 14: Secondary $e^{ \pm}$density in function of primary $\gamma$ and $p$ energy at $10 \mathrm{~m}$ from the core.

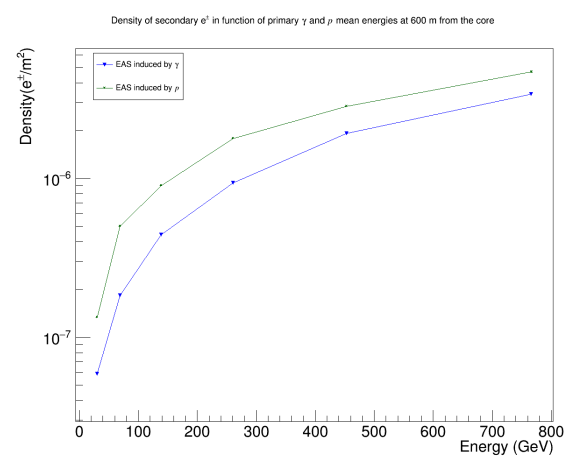

Figure 15: Secondary $e^{ \pm}$density in function of primary $\gamma$ and $p$ energy at $600 \mathrm{~m}$ from the core.

\section{Conclusions}

Obtained results are based on theoretical models of interaction processes between cosmic rays and atmosphere; for the future, when LHAASO and CTA will start to take data, it will be important to compare simulation data to experimental data. It's essential a careful experimental test that, thanks to improvements that LHAASO and CTA will have compared to current Surface Detector (SD) and Imaging Atmosferic Čerenkov Telescope (IACT), it will allow to acquire new knowledge about cosmic rays, their acceleration in galactic and extragalactic sources, propagation in Universe 
and interaction in atmosphere. However, obtained results are important because analysis of $\gamma$ rays showers and proton showers, produced by CORSIKA, it allows us to test teories at the basis of LHAASO and CTA realization, that is thanks to algorithms based on differences between lateral developments of showers in atmosphere, lateral distribution at observation level about charged and neutral particles around shower core, number of $\mu^{ \pm}$, it will be possible to discern $\gamma$ rays showers

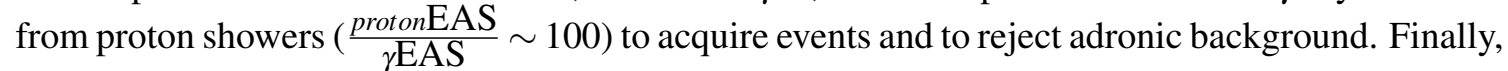
comparing experimental data to obtained mean values of studied physical quantities in function of primary particles energies, it will be possible to estimate the latter.

\section{Acronyms}

CORSIKA Cosmic Ray SImulations for KAscade

CTA Čerenkov Telescope Array

EAS Extensive Air Shower

ED Electron Detector

IACT Imaging Atmosferic Čerenkov Telescope

INFN National Nuclear Physics Institute

LHAASO Large High Altitude Air Shower Observatory

LHAASO-KM2A LHAASO- one Kilometer square extensive air shower Array

LHAASO-WCDA LHAASO- Water Čerenkov Detector Array

LHAASO-WFCTA LHAASO- Wide Field of view Čerenkov Telescope Array

LST Large Size Telescope

MD Muon Detector

MST Medium Size Telescope

SD Surface Detector

SST Small Size Telescope

VHE Very High Energy

\section{References}

[1] F. Casaburo, Rivelazione di fotoni primari nei raggi cosmici di alta energia con apparati Čerenkov imaging e apparati per sciami estesi (Degree thesis, University "La Sapienza" of Rome, 2016)

[2] Multiple Authors, Proposal Large High Altitude Shower Observatory (Chinese Academy of Science Istitute of High Energy of Beijing, 2013) 
[3] F. Ameli et al., LHAASO The Large High Altitude Air Shower Observatory (The LHAASO Collaboration, 2016)

[4] H. He, LHAASO Project: detector design and prototype (The LHAASO Collaboration,2009)

[5] https://portal.cta-observatory.org/SiteAssets/Pages/CTA-Science/ CTA_Special_Issue_own_all.pdf

[6] https://arxiv.org/pdf/1601.04627v1.pdf 